\title{
Strains of Aureobasidium pullulans Can Lower Ochratoxin A Contamination in Wine Grapes
}

\author{
D. V. de Felice, M. Solfrizzo, F. De Curtis, G. Lima, A. Visconti, and R. Castoria
}

First, third, fourth, and sixth authors: Dipartimento di Scienze Animali, Vegetali e dell'Ambiente, Università del Molise, Via F. De Sanctis, 86100, Campobasso, Italy; and second and fifth authors: Istituto di Scienze delle Produzioni Alimentari, C.N.R., Bari, Italy. Accepted for publication 3 July 2008.

\section{ABSTRACT}

De Felice, D. V., Solfrizzo, M., De Curtis, F., Lima, G., Visconti, A., and Castoria, R. 2008. Strains of Aureobasidium pullulans can lower ochratoxin A contamination in wine grapes. Phytopathology 98:1261-1270.

Wine contamination with ochratoxin A (OTA) is due to the attack of wine grapes by ochratoxigenic Aspergillus carbonarius and Aspergillus spp. section Nigri. Four A. pullulans strains, AU14-3-1, AU18-3B, AU342 , and LS30, are resistant to and actively degrade ochratoxin A in vitro. The less toxic ochratoxin $\alpha$ and the amino acid L- $\beta$-phenylalanine were the major degradation products, deriving from the cleavage of the amide bond linking these two moieties of OTA. The same strains were studied further as biocontrol agents of A. carbonarius on wine grapes in labora- tory experiments. Three of the four strains significantly prevented infections by A. carbonarius. Berries pretreated with the biocontrol agents and infected with A. carbonarius contained lower amounts of OTA as compared to the untreated infected control berries. Two of these strains were shown to degrade OTA to ochratoxin $\alpha$ in fresh grape must, but the mechanisms of the decrease of OTA accumulation in infected berries pretreated with the biocontrol agents remain to be elucidated. Assessment of one strain carried out in the vineyard during the growing season of 2006 showed that the tested strain was an effective biocontrol agent, reducing both severity of Aspergillus rots and OTA accumulation in wine grapes. To our knowledge this is the first report describing the positive influence of biocontrol agents on OTA accumulation in this crop species.
Ochratoxin A (OTA) is a mycotoxin produced by fungal species that belong mainly to the genera Aspergillus (A. ochraceus and A. carbonarius) and Penicillium (P. verrucosum). OTA contains a 7carboxy-5-chloro-8-hydroxy-3,4-dihydro-3R-methylisocoumarin fraction (ochratoxin $\alpha$, OT $\alpha$ ) that is linked to the amino acid L- $\beta$ phenylalanine by a carboxy-peptide bond, and is a highly toxic and widespread mycotoxin. This compound is possibly carcinogenic to humans and has been placed in Group 2B by the International Agency for Research on Cancer (32). It has been associated to the Balkan Endemic Nephropathy and to urinary tract tumors in humans $(29,51)$ and it has been shown to be responsible for an endemic porcine nephropathy in northern Europe (38). In addition to its nephrotoxic, teratogenic, and immunosuppressive properties, OTA has been reported to have possible neurotoxic activity (9).

OTA occurs on a wide range of food commodities such as cereals, coffee, cocoa beans, spices, dried fruits, and beer, and since 1996, it has also been detected in grape juice and wine (65). Carry-over from contaminated feedstuffs has resulted in the detection of OTA in human and animal tissues and biological fluids (liver, kidneys, milk, and blood) $(10,11,27,35,46,56)$. Conversely, this mycotoxin is rapidly metabolized by microorganisms colonizing the rumen of cows and sheep by the hydrolytic cleavage of the peptide bond $(30,49)$. This cleavage leads to the separation of L- $\beta$-phenylalanine from the isocoumarin moiety OT $\alpha$ (Fig. 1), which has been shown to be non-nephrotoxic and much less toxic than OTA $(20,24,41,62,64)$.

Although cereals and cereal-based products normally account for 50 to $80 \%$ of the average intake of OTA by consumers, red wine is considered to be another major source of OTA intake in Europe (15). Therefore, maximum tolerable levels for this myco-

Corresponding author: R. Castoria; E-mail address: castoria@unimol.it

doi:10.1094/PHYTO-98-12-1261

(C) 2008 The American Phytopathological Society toxin have been established by the European Commission both in this beverage and in grape juice $(2.0 \mu \mathrm{g} / \mathrm{kg})$ and dried vine fruits $(10.0 \mu \mathrm{g} / \mathrm{kg}$ ) (18). The fungus mainly responsible for OTA contamination of wine grape is A. carbonarius, which belongs to Aspergillus section Nigri, which includes other OTA-producing species that can also attack wine grape (4). A. carbonarius preferentially infects berries through the damaged skin and is the main OTA producer on grapevine since veraison (4). A. carbonarius and OTA can also affect wine grapes during the time interval that usually does not exceed the 24 to $36 \mathrm{~h}$ that commonly elapse from harvest to the beginning of the wine-making process, when bunches of grapes that are detached from the vine lose the protection that the intact plant system has (7). Therefore, the control of $A$. carbonarius both in the field and in the brief time interval between the harvest and the wine-making process is important in order to limit contamination of wine with OTA.

The most effective methods to lower mycotoxin contamination rely on the prevention of the attack of mycotoxigenic fungi, but this approach may not be sufficient to avoid the risk of mycotoxin intake with the diet. Decontamination and/or detoxification methods are also being proposed at a post-production level $(2,15$, 54). OTA levels in wine are effectively lowered by adsorption with fining agents, but these affect the organoleptic quality of the final product $(5,12)$. The integration of preventive measures with the detoxification of OTA to less toxic compounds could be an effective strategy to lower the contamination of wine. A promising approach for preventing fungal attack on agricultural crops is based on the use of biocontrol agents (BCAs), beneficial microorganisms known as nonpathogenic to humans. Although biodegradation of OTA by rumen protozoa $(25)$, bacteria $(31,60)$, and several fungal species has been reported $(1,6,36,58)$, this ability has not been investigated in the case of microbes that serve as BCAs. BCAs act at a preventive level and, if effective against mycotoxigenic fungi, they could prevent mycotoxin biosynthesis and display a potential for mycotoxin degradation. Recently, Castoria et al. (14) demonstrated that the BCA Rhodotorula 
glutinis strain LS11 metabolizes the mycotoxin patulin in vitro. While reducing infections of the mycotoxigenic fungus Penicillium expansum, it also lowers the accumulation of the mycotoxin in infected apples under storage.

Aureobasidium pullulans is an ascomycetous yeastlike fungus that has found many industrial applications, such as production of antibiotics (57) and of pullulan, a complex polysaccharide used for coating and packaging of food (61). It is a common inhabitant of the phylloplane (45), and the main species isolated from immature, mature, and both damaged and undamaged wine grapes on which it appears to persist until harvest (52). A few strains of A. pullulans have been described as effective BCAs in different pathosystems, including diseases that affect grapes $(13,33,34$, 39,43).

The aim of the present study was to assess the capability of $A$. pullulans strains to degrade OTA, and to assess the influence of these microbial agents on accumulation of OTA in wine grape berries, where the biosynthesis of this mycotoxin by A. carbonarius takes place. Following an initial in vitro screening of 12 strains of A. pullulans, four strains (AU14-3-1, AU18-3B, AU342 , and LS30) were selected as the strains yielding the greatest decrease of toxin concentration. These four strains were further examined for their ability to protect wine grape berries from $A$. carbonarius infection and their effects on the accumulation of OTA in pathogen-infected berries in laboratory-scale experiments. A preliminary report has been published earlier (22). Assessment of biocontrol activity of one (LS30) of the four strains to Aspergillus rots and OTA accumulation in wine grapes was also carried out in the vineyard.

\section{MATERIALS AND METHODS}

Chemicals used. A commercial standard of OTA was pur${ }^{\circ}$ chased from Sigma-Aldrich (Milan, Italy). A stock solution of OTA $1 \mathrm{mg} / \mathrm{ml}$ in toluene/acetic acid (99:1, vol/vol) was prepared. Immediately prior to use, working solutions used in the experiments were prepared by drying aliquots of the stock solution under a nitrogen stream, and by redissolving them in acetonitrile/water/acetic acid (99:99:2, vol/vol/vol). The solvent mixture was also used as mobile phase for high-performance liquid chromatography (HPLC) analyses. All solvents employed were bi-distilled or HPLC grade. All solvents and reagents were obtained from Sigma-Aldrich (Milan, Italy). Nutrient broth and yeast extract powder were purchased from Oxoid Ltd. (Basingstoke, Hampshire, England), dextrose from Carlo Erba Reagenti S.p.a. (Milan, Italy). The OT $\alpha$ standard was obtained by enzymatic hydrolysis of OTA with carboxypeptidase A from bovine pancreas (EC 3.4.17.1) (Sigma-Aldrich, Milan, Italy), according to Hassen et al. (28).

Microorganisms used. Twelve A. pullulans strains (Table 1) were tested in vitro in a preliminary screening for their resistance to OTA and their capability of decreasing OTA concentration in their growth medium. Eleven strains were from the culture collection of the Dipartimento di S.A.V.A. of the University of Molise and strain FSE L2 was a gift from L. Caputo, Istituto di Scienze delle Produzioni Alimentari, Bari, Italy. Strain LS30 had previously been sent to CBS (Centraalbureau voor Schimmelcultures, The Netherlands) for identification that was carried out on a morphological basis (accession no. CBS 110902), the other eleven strains were also identified as A. pullulans on a morphological basis (colony color, shape, and microscopic observations). Strains AU18-2A, AU24, AU28, AU29, AU33, AU34-2, and LS30 had been characterized at a genomic level through fluorescent amplified fragment length polymorphism analysis (21). In subsequent experiments four A. pullulans strains were used: AU14-3-1, AU18-3B, AU34-2, and LS30. Strain LS30 had previously been shown to have biocontrol activity to different pathogens on a variety of crop species and had also been characterized for its modes of action in biocontrol $(13,43)$. The OTA-producing strain A1102 of $A$. carbonarius that was used in the present biocontrol trials was kindly provided by $\mathrm{P}$. Battilani, Università Cattolica del Sacro Cuore, Piacenza, Italy.

In vitro degradation of OTA by strains of $A$. pullulans. The four strains used in this work were chosen following a preliminary thin layer chromatography (TLC)-based screening of $12 \mathrm{~A}$. pullulans strains (see previously) for their in vitro capability of resisting OTA and lowering its initial concentration of $2.0 \mu \mathrm{M}$ (corresponding to $0.8 \mu \mathrm{g} / \mathrm{ml}$ ) in Lilly-Barnett growth medium (LiBa, $10.0 \mathrm{~g}$ of D-glucose, $2.0 \mathrm{~g}$ of L-asparagine, $1.0 \mathrm{~g}$ of $\mathrm{KH}_{2} \mathrm{PO}_{4}, 0.5 \mathrm{~g}$ of $\mathrm{MgSO}_{4} \times 7 \mathrm{H}_{2} \mathrm{O}, 0.01 \mathrm{mg}$ of $\mathrm{FeSO}_{4} \times 7 \mathrm{H}_{2} \mathrm{O}$,

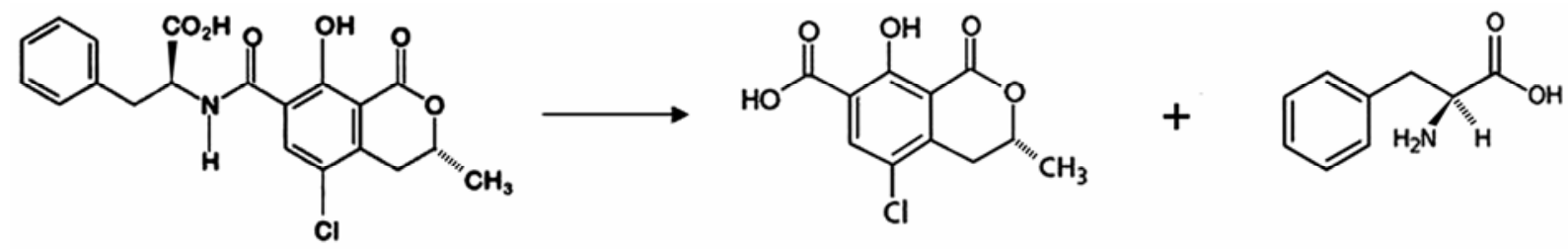

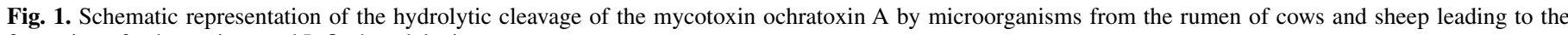
formation of ochratoxin $\alpha$ and L- $\beta$-phenylalanine.

TABLE 1. Source and site of isolation of the Aureobasidium pullulans strains used in this study

\begin{tabular}{|c|c|c|c|}
\hline Strain $^{\mathrm{a}}$ & Isolation source & Geographical site & Reference source \\
\hline AU14-3-1 & Apple leaves & Larino (Campobasso, Italy) & This study \\
\hline AU18-1 & Apricot leaves & Lucera (Foggia, Italy) & This study \\
\hline AU18-2A & Apricot flowers & Lucera (Foggia, Italy) & 21 \\
\hline AU18-3B & Plum fruits & Lucera (Foggia, Italy) & This study \\
\hline AU22-1 & Apple leaves & S. Bartolomeo in Galdo (Benevento, Italy) & This study \\
\hline AU24 & Plum fruits & S. Bartolomeo in Galdo (Benevento, Italy) & 21 \\
\hline AU28 & Grapevine leaves & S. Giuliano (Campobasso, Italy) & 21 \\
\hline AU29 & Apple leaves & S. Giuliano (Campobasso, Italy) & 21 \\
\hline AU33 & Lemon tree leaves & Collepasso (Lecce, Italy) & 21 \\
\hline AU34-2 & Grapevine leaves & Collepasso (Lecce, Italy) & 21 \\
\hline FSE L2 & Rose leaves & Bari (Italy) & This study \\
\hline LS30 & Apple fruits & Sessa Aurunca (Caserta, Italy) & CBS n. $110902,13,21,43$ \\
\hline
\end{tabular}

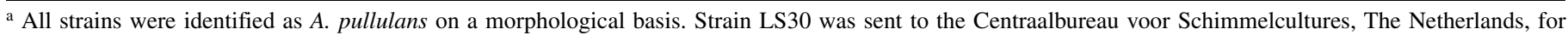
identification, which was also carried out on a morphological basis. 
$8.7 \mathrm{mg}$ of $\mathrm{ZnSO}_{4} \times 7 \mathrm{H}_{2} \mathrm{O}, 3.0 \mathrm{mg}$ of $\mathrm{MnSO}_{4} \times \mathrm{H}_{2} \mathrm{O}, 0.1 \mathrm{mg}$ of Biotin, and $0.1 \mathrm{mg}$ of Thiamine) (42). The strains were grown as later described. At the end of the incubation, cell cultures were centrifuged at 4,000 rpm and 40- $\mu \mathrm{l}$ aliquots of culture supernatants were spotted directly onto silica gel 60 TLC plates (Whatman, Maidstone, UK). The plates were developed in toluene/ethyl acetate/formic acid (5:4:1, vol/vol/vol). Recovery of OTA from the growth medium was determined by visually assessing the intensity of the corresponding spot under UV light $(\lambda=365 \mathrm{~nm})$. Experiments were performed three times. The strains AU14-3-1, AU18-3B, AU34-2, and LS30 that caused a reduction of OTA concentration greater than $50 \%$, as compared to negative control (OTA incubated in the noninoculated medium) and to initial OTA concentration (data not shown), were chosen for the following experiments.

Cells of each of the four strains AU14-3-1, AU18-3B, AU34-2, and LS30 were scraped from a petri dish, added to $50 \mathrm{ml}$ of nutrient yeast dextrose broth medium (NYDB, $8 \mathrm{~g}$ of nutrient broth, $5 \mathrm{~g}$ of yeast extract, and $10 \mathrm{~g}$ of dextrose in 1 liter of water) (Oxoid Ltd, Basingstoke, Hampshire, UK) and grown overnight at $23^{\circ} \mathrm{C}$. Cultures of each strain at the same concentration $\left(1 \times 10^{5}\right.$ colony forming units $[\mathrm{CFU}] / \mathrm{ml}$ ) were prepared into three flasks each containing $50 \mathrm{ml}$ of LiBa medium amended with $40 \mu \mathrm{l}$ of OTA $1 \mathrm{mg} / \mathrm{ml}$ in acetonitrile/water/acetic acid (99:99:2, $\mathrm{vol} / \mathrm{vol} / \mathrm{vol}$ ) (final OTA concentration $0.8 \mu \mathrm{g} / \mathrm{ml}$, corresponding to $2.0 \mu \mathrm{M}$ ), and grown for 6 days at $23^{\circ} \mathrm{C}$ on a rotary shaker at 160 rpm. Two of the four strains, AU14-3-1 and AU18-3B, were also grown on fresh sterile grape must obtained by pressing wine grape bunches (cv. Montepulciano), which had been previously sterilized as later described. The liquid obtained was filtered through a sterile strainer and underwent heat treatment at $100^{\circ} \mathrm{C}$ for $30 \mathrm{~min}$. After spontaneous deposition of precipitates on the bottom of the flask, residual liquid must was transferred to new sterile flasks with sterile syringes prior to inoculation. Controls consisted of noninoculated media (LiBa and must), amended with OTA, and of the A. pullulans strains grown in the same media, amended with $40 \mu \mathrm{l}$ acetonitrile/water/acetic acid (99:99:2, $\mathrm{vol} / \mathrm{vol} / \mathrm{vol}$ ) only. Aliquots of cultures were plated on petri dishes with nutrient yeast dextrose agar medium (NYDA, $8 \mathrm{~g}$ of nutrient broth, $5 \mathrm{~g}$ of yeast extract, $10 \mathrm{~g}$ of dextrose, and $20 \mathrm{~g}$ of agar in 1 liter water) after dilution on day 1, 2, 3, 4, 5, and 6 of incubation to assess the growth of each strain over time. At the same time points, the quantities of OTA and OT $\alpha$ were also analyzed by HPLC. The experiment was performed three times and each experiment consisted of three replicates. Data from the experiments were pooled, since they were similar in the three repetitions.

Biocontrol activity of $A$. pullulans strains on wine grape berries in laboratory-scale experiments. Healthy bunches of mature wine grape cv. Montepulciano used in this study were purchased from local farmers. Grapes had undergone precocious treatment (at the pre-bunch closure stage) only with Switch (a mixture of the nonsystemic fungicides cyprodinil and a contact fungicide fludioxonil) and copper-based fungicides (contact fungicides) from veraison to ripening. Prior to treatment with microorganisms, mature wine grape berries were detached from the bunches by cutting the grape stalk at about $1 \mathrm{~cm}$ from each berry in order to prevent dessication. Detached berries were immediately sterilized for $2 \mathrm{~min}$ in a $1 \%$ sodium hypochlorite solution $(\mathrm{pH} 11.5)$, washed twice thoroughly with sterile water to eliminate possible fungicide residues, and dried externally on absorbent paper. Intact berries were then wounded at three equidistant points halfway from the point of emergence of the grape stalk, and treated by letting them shake in suspensions $\left(10^{8}\right.$ cell $\left./ \mathrm{ml}\right)$ of the four A. pullulans strains (LS30, AU14-3-1, AU18-3B, and AU34-2) for $5 \mathrm{~min}$ at $80 \mathrm{rpm}$. Berries were then sprayed with a suspension of $A$. carbonarius A1 102 conidia that had previously been prepared as follows: A. carbonarius was grown on potato dextrose agar (PDA, Oxoid Ltd., Basingstoke, Hampshire, UK) and, after 15 days of incubation at $28^{\circ} \mathrm{C}$, the mycelium and the conidia were scraped from the plate and transferred to a Falcon tube containing sterile distilled water. The tube was stirred on a vortex, filtered, and centrifuged at 3,200 $\mathrm{g}$ for $10 \mathrm{~min}$. The pellet was resuspended in sterile distilled water and the suspension adjusted to $5 \times 10^{4}$ conidia/ml. Each single berry was then sprayed with $35 \mu \mathrm{l}$ of the conidial suspension of $A$. carbonarius. After spraying, berries were placed, well separated from each other, on a grate previously disinfected with ethanol. Grates were placed into disinfected plastic boxes containing $300 \mathrm{ml}$ of water to achieve $100 \%$ relative humidity $(\mathrm{RH})$. Boxes were hermetically sealed and incubated at $24 \pm 1^{\circ} \mathrm{C}$. Infections of wounds were assessed directly or by means of a stereomicroscope. Percentages of infected wounds were recorded on day 1,2,3, 4, and 6 of incubation. Each treatment consisted of five replicates of 20 berries. The control consisted of the same number of wounded berries, not pretreated with the A. pullulans strains, but sprayed with a suspension of conidia of $A$. carbonarius A1102. Experiments were performed three times, and the data were pooled, since they were similar in the three repetitions. Percentage values were converted into Bliss angular values (arcsine $\sqrt{ } \%$ ) prior to statistical analysis. Transformed data were submitted to factorial analysis of variance (ANOVA) by using the PROC general linear models (GLM) of SPSS (version 16.0 for Windows; SPSS Inc., Chicago, IL). Means were separated by the least significant difference (LSD) test $(P<0.01)$.

Survival of $A$. pullulans strains on infected berries in laboratory-scale experiments. Two infected berries from each replicate were randomly collected among all the berries showing symptoms of infection after 6 days of incubation. Berries were washed for $20 \mathrm{~min}$ with $20 \mathrm{ml}$ of sterile water on a rotary shaker at $160 \mathrm{rpm}$, and $100 \mu \mathrm{l}$ of these washes were plated after dilution on petri dishes with NYDA medium to assess the presence of the four A. pullulans strains on grape berries infected by A. carbonarius. Experiments were performed three times and the data were pooled, since they were similar in the three repetitions.

Determination of OTA and $\alpha$ in culture filtrates of $A$. pullulans strains grown in Lilly-Barnett medium, grape must, and in infected grape berries from laboratory-scale experiments. Culture filtrates of A. pullulans strains grown in LiBa medium in the presence of OTA were appropriately diluted and analyzed by HPLC/fluorescence detection to quantify OTA and OT $\alpha$. Sample preparation and a cleanup step were necessary for chemical analysis of inoculated grape berries and must. Before determining OTA and OT $\alpha$ in grape berries and must, the recovery efficiency of the extraction procedures later described was assessed by spiking OTA- and OT $\alpha$-free berry puree and must with known amounts of the two compounds. The overall mean OTA recovery from berry puree and must were 107.1 and $109.6 \%$, respectively; the overall mean for OT $\alpha$ recoveries from berry puree and must were 92.9 and $99.6 \%$, respectively. Values of OTA and OT $\alpha$ in the experiments below were corrected accordingly.

The same grape berries used in biocontrol assays from laboratory-scale experiments were sampled for comparing the accumulation of OTA and OT $\alpha$ when infection by A. carbonarius occurred in the presence and in the absence of the biocontrol agents. At day 6, infected berries (i.e., only berries that showed symptoms of infection) from the different treatments were randomly collected and homogenized with an Ultra-Turrax (IKAWerke GmbH \& Co. KG, Staufen, Germany) at 14,000 rpm. Samples of $5 \mathrm{~g}$ were added to $10 \mathrm{ml}$ of a water solution with $1 \%$ (wt/vol) polyethylene glycol and 5\% (wt/vol) sodium hydrogen carbonate, mixed vigorously for $3 \mathrm{~min}$ and centrifuged at 4,000 $\mathrm{rpm}$ for $10 \mathrm{~min}$. The supernatants were filtered through Whatman GF/A glass microfibre filters in order to obtain a clear juice. Aliquots of $3 \mathrm{ml}$ from these extracts were loaded on $\mathrm{C}_{18}$ Sep-Pak RC 500-mg cartridges (Waters, Milford, MA) previously conditioned with $4 \mathrm{ml}$ of methanol, $4 \mathrm{ml}$ of water, and $2 \mathrm{ml}$ of a 
$5 \%$ sodium hydrogen carbonate solution. $\mathrm{C}_{18}$ cartridges were washed with $2 \mathrm{ml}$ of $0.1 \mathrm{M}$ phosphoric acid and $2 \mathrm{ml}$ of water. OT $\alpha$ was eluted with $4 \mathrm{ml}$ of ethyl acetate/methanol/acetic acid (95:5:0.5, vol/ $\mathrm{vol} / \mathrm{vol})$, whereas OTA was eluted with $2 \mathrm{ml}$ of acetonitrile/acetic acid $(98: 2, \mathrm{vol} / \mathrm{vol})$ in a separate vial. The purified extract containing OT $\alpha$ was dried under a nitrogen stream, redissolved with the purified extract containing OTA, and further diluted with $1 \mathrm{ml}$ of distilled water. Aliquots of $50 \mu \mathrm{l}$ of the purified extracts were analyzed with an HPLC with fluorometric detection. The HPLC apparatus was an Agilent 1100 series equipped with a $\mathrm{G} 1312 \mathrm{~A}$ binary pump, $\mathrm{G} 1313 \mathrm{~A}$ autosampler, $\mathrm{G}$ $1316 \mathrm{~A}$ column thermostat set at $30^{\circ} \mathrm{C}, \mathrm{G} 1321 \mathrm{~A}$ spectrofluorometric detector set at $333 \mathrm{~nm}\left(\lambda_{\mathrm{ex}}\right)$ and $460 \mathrm{~nm}\left(\lambda_{\mathrm{em}}\right)$, and Agilent Chemstation G 2170AA Windows 2000 operating system (Agilent, Waldbronn, Germany). The separations were performed with an Xterra $\mathrm{C}_{18}$ column $(150 \times 4.6 \mathrm{~mm}-5 \mu \mathrm{m})$ preceded by a guard column with the same packing material (Waters, Milford, MA). The mobile phase was an isocratic mixture of acetonitrile/water/acetic acid (99:99:2, vol/vol/vol) eluted at a flow rate of $1.0 \mathrm{ml} / \mathrm{min}$. Mixed solutions of enzymatically-produced OT $\alpha$ and commercial OTA in the mobile phase were injected, and peak areas were determined to generate calibration curves for quantitative analyses. Procedures of extraction and analysis of OTA and OT $\alpha$ in samples of $5 \mathrm{ml}$ must were as described previously for grape berries, with the omission of the homogenization step.

Selected culture filtrates of $A$. pullulans strains were analyzed by liquid chromatography-mass spectrometry (LC-MS) to confirm the identity of OT $\alpha$ and phenylalanine as the OTA breakdown products. LC-MS analyses were performed on an MS/MS QTrap system from Applied Biosystems (Foster City, CA), equipped with an ESI (electrospray ionization) interface and an 1100 series micro-LC system comprising a binary pump and a micro-autosampler from Agilent Technologies (Waldbronn, Germany). The column was a Synergy hydro $(150 \times 2 \mathrm{~mm}, 4 \mu \mathrm{m}$ particle size). Two isocratic mixtures of acetonitrile/water containing $0.1 \%$ acetic acid (50:50, vol/vol) at a flow rate of $0.2 \mathrm{ml} / \mathrm{min}$ were used as the mobile phase: $1 \%$ acetonitrile was pumped for $5 \mathrm{~min}$ and followed by $60 \%$ acetonitrile for $15 \mathrm{~min}$. The mass spectrometer was set in the selected-ion monitoring mode using $m / z$ 402.0 [OTA-H] ${ }^{-}$for OTA, $m / z$ 255.0 [OT $\left.\alpha-\mathrm{H}\right]^{-}$ for OT $\alpha$ and $m / z, 166.0[\mathrm{Phe}+\mathrm{H}]^{+}$for phenylalanine, which had retention times of $14.85,10.75$, and $9.78 \mathrm{~min}$, respectively.

Biocontrol activity of $A$. pullulans strain LS30 in the vineyard. Strain LS30 that had previously been shown to have biocontrol activity to different pathogens on a variety of crop species $(13,43)$ was also tested for its biocontrol activity to Aspergillus spp. on wine grapes in the vineyard during the growing seasons 2006 and 2007.

The vineyard, which consisted of vines (cv. Montepulciano) trained with the overhead arbor system, was located in Abruzzo, a region on the Adriatic coast of Central-Eastern Italy. The cell suspension of the antagonist was prepared as follows: LS30 cells were scraped from a petri dish containing NYDA, added to $50 \mathrm{ml}$ of NYDB, and grown on a rotary shaker at $150 \mathrm{rpm}$ overnight at $23^{\circ} \mathrm{C}$. The cell suspension was then added to 5 liters of fresh $\mathrm{NYDB}$ and grown at $150 \mathrm{rpm}$ for $48 \mathrm{~h}$ at $23^{\circ} \mathrm{C}$. The culture was centrifuged at 4,000 rpm for $15 \mathrm{~min}$, the pellet was resuspended in tap water, and the concentration adjusted to $5 \times 10^{7} \mathrm{CFU} / \mathrm{ml}$ using an hematocymeter. The antagonist suspension was applied by means of a motor-driven back-pack sprayer (delivering approximately $10 \mathrm{hl} / \mathrm{ha}$ ) at the following phenological stages: bunch preclosure, early veraison, and 20 days before harvest. Field trials were arranged in a completely randomized block design with four replicates per treatment and six plants per each replicate. The control consisted of completely untreated plants.

The influence of LS30 on the incidence (\% of infected bunches) and severity (\% of infected berries per bunch) of decay caused by Aspergillus spp. was evaluated just before harvest.
Percentage values of disease incidence and severity were converted into Bliss angular values (arcsine $\sqrt{ } \%$ ) prior to statistical analysis and submitted to the Student's $t$ test.

At harvest, in order to verify the presence and quantify the population of potentially ochratoxigenic Aspergillus spp. section Nigri in treated and untreated plots, 24 bunches per replicate (four bunches per plant) were collected in sealed plastic bags and kept in a portable refrigerator for their transport to the laboratory. The bunches of each replicate were pooled and homogenized by means of an Ultra-Turrax blender at $14,000 \mathrm{rpm}$. Three subsamples of $10 \mathrm{ml}$ of the homogenate were withdrawn and used for both Aspergillus isolation and quantitative determination of OTA. Aspergillus species were isolated by plating $100 \mu \mathrm{l}$ of serial dilutions of the grape homogenate into petri dishes containing the semiselective medium Dichloran Rose Bengal Chloramphenicol (DRBC) (37). Plates were incubated for 5 to 7 days at $25^{\circ} \mathrm{C}$, and Aspergillus species were macroscopically and microscopically identified according to rules and descriptions provided by Raper and Fennell (53).

Determination of OTA in vineyard samples. The same homogenized subsamples used for isolation and identification of Aspergillus spp. were also analyzed by HPLC to determine the influence of strain LS30 on OTA accumulation in grapes. OTA was extracted and analyzed as described previously. Analyses were performed twice. Levels of OTA contamination were expressed as nanograms per gram of grape and submitted to the Student's $t$ test.

\section{RESULTS}

Degradation of OTA by strains of $A$. pullulans in LillyBarnett medium. The in vitro preliminary screening, performed to assess the capability of $12 \mathrm{~A}$. pullulans strains to decrease OTA concentration in LiBa, showed that strains AU14-3-1, AU18-3B, AU34-2, and LS30 caused the most remarkable decrease intensity of OTA spots $(\mathrm{Rf}=0.78)$ on TLC plates, and the parallel appearance of a new spot with $\mathrm{Rf}=0.58$, corresponding to OT $\alpha$ (data not shown).

The influence of OTA on the growth of the best-performing strains identified in the preliminary screening, AU14-3-1, AU183B, AU34-2, and LS30 over 6 days in LiBa is shown in Figure 2. The presence of OTA did not affect the growth pattern of the four A. pullulans strains. At all time points, the cell density in the absence of OTA was not significantly different from that in the presence of the mycotoxin (Fig. 2A through D). Figure 2 also reports the quantitative results of OTA and OT $\alpha$ in time-course HPLC analyses of the same in vitro experiments for all the four strains. Figure 3 shows typical chromatograms on which the graphs of OTA and OT $\alpha$ in Figure 2A are based using biodegradation by strain AU14-3-1 as an example. All the four strains caused a dramatic reduction of OTA concentration in the growth medium (Fig. 2A through D). The observed decreases of OTA concentration paralleled the time-course increase of OT $\alpha$, whose identity was confirmed by comparing its retention time $\left(R_{t} 2.9\right.$ min) with the one of standard OT $\alpha$ obtained by enzymatic hydrolysis of commercial OTA. The identity of OT $\alpha$ was further confirmed by LC-MS analyses, which also identified L- $\beta$ phenylalanine as the other breakdown product (data not shown). On the sixth day of the experiment, the OTA concentrations ranged from $0.50 \mu \mathrm{mol} / \mathrm{liter}$ for strain LS30 to $0.19 \mu \mathrm{mol} / \mathrm{liter}$ for strain AU34-2. The OT $\alpha$ concentrations on day 6 ranged from $1.89 \mu \mathrm{mol} / \mathrm{liter}$ for strain AU14-3-1 to $1.85 \mu \mathrm{mol} / \mathrm{liter}$ for strain AU34-2. HPLC analyses of cell pellets that had been extracted with a mixture of acetonitrile/water (60:40, vol/vol) showed only traces of OTA, which demonstrates that there was no OTA adsorption to the cells of the four strains.

Biocontrol activity of $A$. pullulans strains on wine grape berries in laboratory-scale experiments. The level of antago- 
nism of the four biocontrol agents AU14-3-1, AU18-3B, AU34-2, and LS30 to A. carbonarius A1102 on grape wine berries in laboratory-scale experiments is shown in Figure 4. Strains AU143-1, AU18-3B, and LS30 displayed a good antagonistic activity, significantly lowering the levels of infections by A. carbonarius, whereas AU34-2 was less effective in protecting the wine grape berries. On day 1 , when almost $10 \%$ of wounds were infected by $A$. carbonarius in control berries nonpretreated with the $A$. pullulans strains, no wounds were infected by the fungus in all BCA-pretreated berries. On day 2, when $67 \%$ of untreated control wounds were infected by A. carbonarius, strains AU14-3-1 and AU18-3B completely prevented infection of wounded berries,

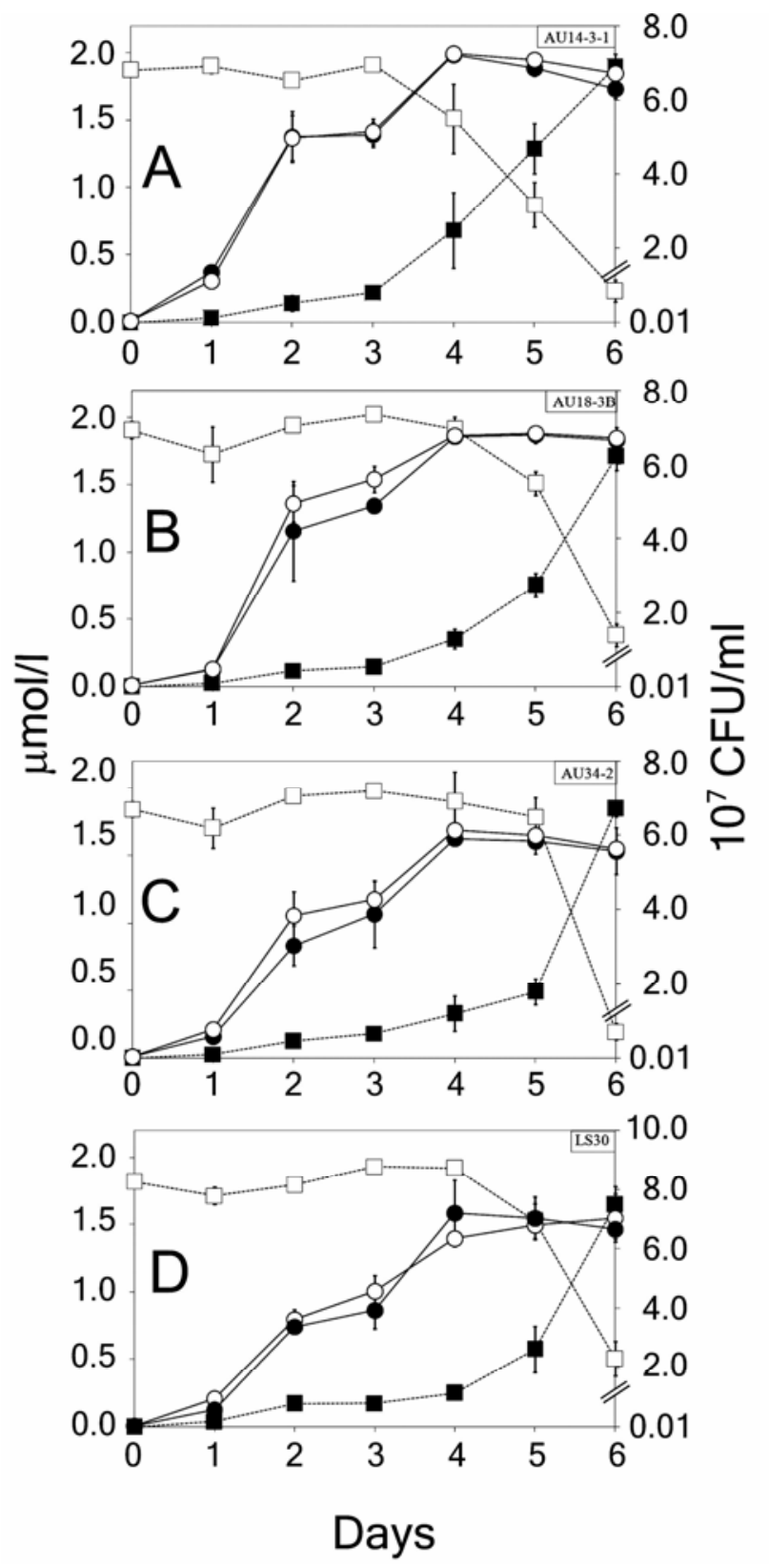

Fig. 2. Ochratoxin A (OTA) degradation and growth of Aureobasidium pullulans strains A, AU14-3-1, B, AU18-3B, C, AU34-2, and D, LS30 during 6 days of incubation at $23^{\circ} \mathrm{C}$ in Lilly-Barnett medium. Dotted lines describe the levels of OTA ( $\square)$ and ochratoxin $\alpha(\mathrm{OT} \alpha, \mathbf{\square})$ in the presence of $A$. pullulans strains. Full lines describe the growth of the same A. pullulans strains as above in the presence $(O)$ and in the absence $(-)$ of $2.0 \mu \mathrm{M}$ OTA. The initial OTA concentration $(2.0 \mu \mathrm{M})$ in the absence of the A. pullulans strains remained unchanged over the entire duration of the experiments. Values are the means \pm standard deviation $(n=9,3$ measurements in three experiments). whereas only 2.2 and $1.1 \%$ of wounds were infected when strains AU34-2 and LS30 were used as protectants, respectively. After 3 days of incubation, $100 \%$ of the wounds of the untreated control berries were infected, whereas only $0,2.2,6.7$, and $31.1 \%$ of the wounds were infected in berries that had been pretreated with strains AU14-3-1, AU18-3B, LS30, and AU34-2, respectively. Protection of grape berries by the same strains was still evident on day 4 , when $3.3,2.2$, and $24.4 \%$ of the wounds were infected when pretreated with strains AU14-3-1, AU18-3B, and LS30, respectively, whereas AU34-2 was less effective, allowing 64.4\% of the wounds to become infected. On day 6 , the level of protection provided by the four strains showed a clear decrease:
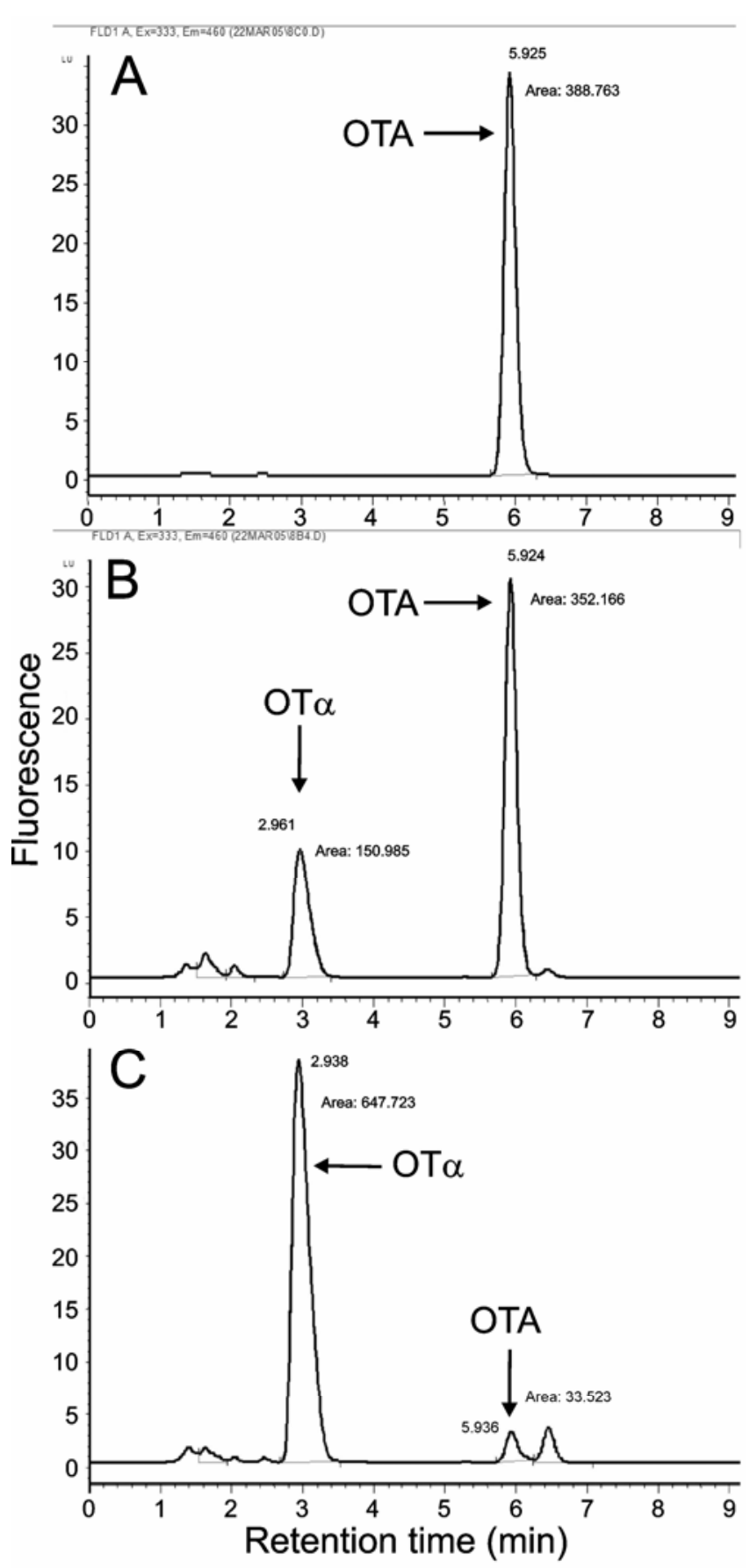

Fig. 3. High-performance liquid chromatography (HPLC) chromatograms of culture extracts of Aureobasidium pullulans strain AU14-3-1 at time A, 0, B, after 4 days, and $\mathbf{C}, 6$ days of incubation at $23^{\circ} \mathrm{C}$ in Lilly-Barnett medium in the presence of $2.0 \mu \mathrm{M}$ ochratoxin A (OTA). The retention time of OTA is $5.92 \mathrm{~min}$ and the retention time of ochratoxin $\alpha(\mathrm{OT} \alpha)$ is $2.95 \mathrm{~min}$. 
$64.4 \%$ of the wounds were infected by $A$. carbonarius in berries pretreated with strains AU14-3-1 and AU18-3B, and 75.6 and 91.1\% were infected when pretreated with LS30 and AU34-2, respectively, with no significant difference of protection achieved by the latter strain, as compared to the untreated control. Factorial analysis showed that the effects of the A. pullulans strains and of time (days after the treatments) were highly significant $(P<$ 0.001 ), although their interaction (treatment $\times$ time) was not statistically significant $(P=0.275)$ (Table 2$)$.

Levels of OTA and $\alpha$ in $A$. carbonarius-infected berries from laboratory-scale experiments. Figure 5 shows the quantitative results of HPLC analyses of OTA and OT $\alpha$ in the infected berries from the biocontrol experiments, 6 days after inoculation with $A$. carbonarius. The concentration of OTA in infected berries that had been pretreated with A. pullulans AU14-3-1 and AU18$3 \mathrm{~B}$ was significantly lower than in the control, i.e., in the infected berries not pretreated with the BCAs: pretreatments with AU14-31 and AU18-3B resulted in 75.5\% (109.1 ng/g) and 70.9\% $(129.3 \mathrm{ng} / \mathrm{g})$ reductions of OTA contamination, respectively, compared with that recorded in the control $(445.5 \mathrm{ng} / \mathrm{g})$. In the case of the other two strains, LS30 and AU34-2, the contamination was 269.4 and $299.2 \mathrm{ng} / \mathrm{g}$, respectively, but not statistically different from that recorded in the control treatment. Low levels of OT $\alpha$ were recorded in all treatments, ranging from $17.9 \mathrm{ng} / \mathrm{g}$ in the infected berries not pretreated with the BCAs to $9.7 \mathrm{ng} / \mathrm{g}$ for those pretreated with strain AU18-3B.

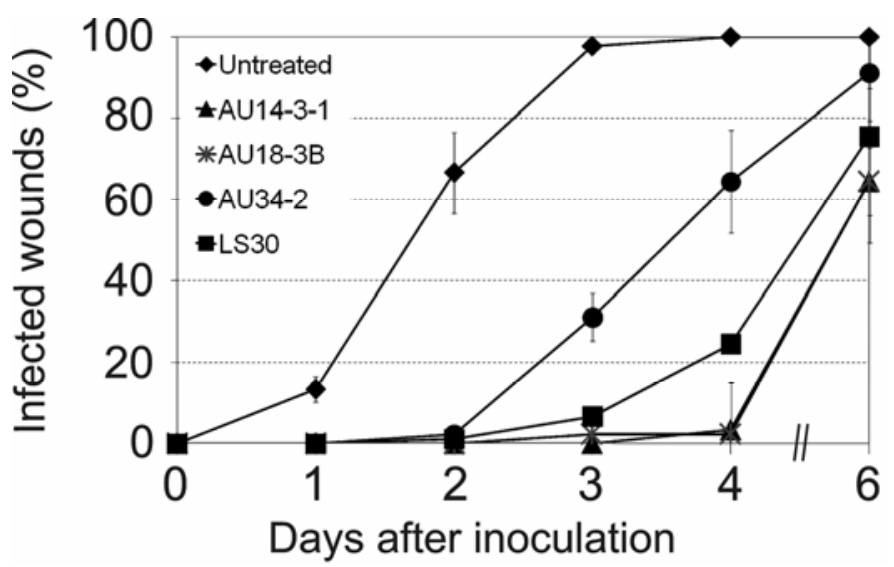

Fig. 4. Biocontrol activity of Aureobasidium pullulans (strains AU14-3-1, AU18-3B, AU34-2, and LS30) on wine grape berries (cv. Montepulciano) inoculated with Aspergillus carbonarius strain A1102. Biocontrol activity was expressed as percentages \pm standard deviation of infected wounds that were recorded after $1,2,3,4$, and 6 days of incubation at $24 \pm 1_{-}^{\circ} \mathrm{C}$. The untreated control consisted of berries that had not been pretreated with the A. pullulans strains prior to inoculation with the fungal pathogen. Symbols at each time point represent mean values \pm standard deviation of three different experiments $(n=9)$. Percentage values were converted into Bliss angular values prior to statistical analysis. Means, based on combined data from three experiments, were separated by the least significant difference (LSD) test $(P<$ $0.001)$. LSD $=29.84 \%$.
Fate of $A$. pullulans strains on $A$. carbonarius-infected berries from laboratory-scale experiments. All of the strains of A. pullulans examined in this study survived in A. carbonariusinfected berries during the course of the laboratory-scale experiments and by 6 days reached average values of $9.9 \times 10^{5}, 4.7 \times$ $10^{6}, 6.4 \times 10^{6}$, and $7.1 \times 10^{6} \mathrm{CFU}$ per berry for strains AU34-2, AU14-3-1, AU18-3B, and LS30, respectively.

Degradation of OTA in grape must by strains AU14-3-1 and AU18-3B. The growth of strains AU14-3-1 and AU18-3B of A. pullulans was not affected by the presence of OTA when grown in freshly prepared must (Fig. 6). Over 6 days, the cell density in the presence of OTA was not significantly different from that in the absence of the mycotoxin (Fig. 6A and B).

Quantitative results for OTA and OT $\alpha$ from time-course HPLC analyses of the same experiments are also shown in Figure 6.

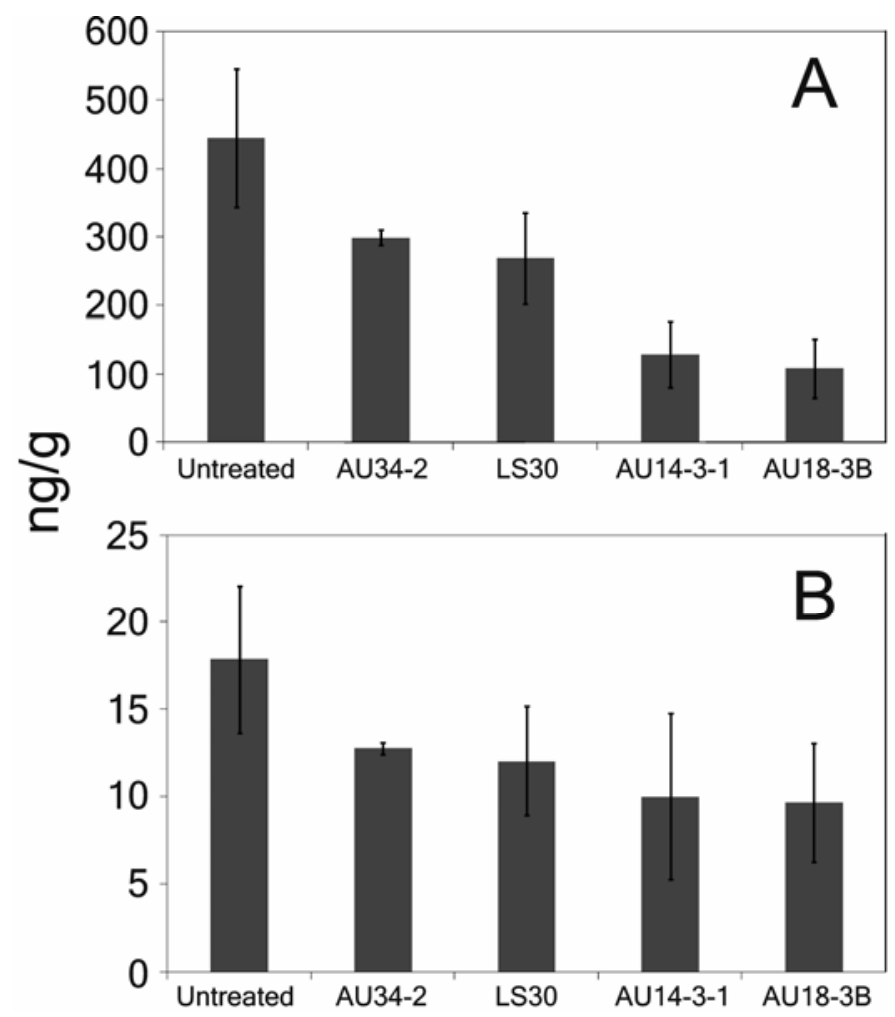

Fig. 5. Concentration of A, ochratoxin A (OTA) and $\mathbf{B}$, ochratoxin $\alpha$ analysed by high-performance liquid chromatography (HPLC) in berries pretreated with Aureobasidium pullulans strains AU14-3-1, AU18-3B, AU34-2, and LS30, and infected by Aspergillus carbonarius strain A1102. The untreated control consisted of berries that had not been pretreated with A. pullulans strains but inoculated with the fungal pathogen. Bars represent mean values \pm standard deviations of three experiments after 6 days of incubation at $24 \pm$ $1{ }^{\circ} \mathrm{C}$. Means, based on combined data from three experiments, were separated by the least significant difference (LSD) test. LSD $=197.37(P=0.020)$ in $\mathbf{A}$; means were not significantly different $(P=0.098)$ in $\mathbf{B}$.

TABLE 2. Analysis of variance of the influence of treatments (AU14-3-1, AU18-3B, AU34-2, and LS30 strains of Aureobasidium pullulans) and time (days after treatments) on percentage of wounds infected by Aspergillus carbonarius

\begin{tabular}{|c|c|c|c|c|c|}
\hline Source of variability & Type III SS ${ }^{\mathrm{a}}$ & df & Means of squares & $F$ & Sig. \\
\hline Corrected model & $57,384,861^{\mathrm{b}}$ & 29 & $1,978,788$ & 5,939 & 0.000 \\
\hline Intercept & $40,508,875$ & 1 & $40,508,875$ & 121,576 & 0.000 \\
\hline Treatment (A) & $10,138,427$ & 4 & $2,534,607$ & 7,607 & 0.000 \\
\hline Time (B) & $39,156,435$ & 5 & $7,831,287$ & 23,503 & 0.000 \\
\hline $\mathrm{A} \times \mathrm{B}$ & $8,089,998$ & 20 & 404,500 & 1,214 & 0.275 \\
\hline Error & $19,991,807$ & 60 & 333,197 & & \\
\hline Total & $117,885,542$ & 90 & & & \\
\hline Corrected total & $77,376,667$ & 89 & & & \\
\hline
\end{tabular}

a $\mathrm{SS}=$ sum of squares.

${ }^{\mathrm{b}} \mathrm{R}$ squared $=0,742$ (adjusted $\mathrm{R}$ squared $=0,617$ ). 
Both strains, AU14-3-1 and AU18-3B, caused a significant reduction of OTA concentration in must compared with the noninoculated control (Fig. 6A and B). As in the case of growth in LiBa (Fig. 2), the decrease of OTA concentration paralleled the time-course increase of OT $\alpha$. On the sixth day of the experiment,

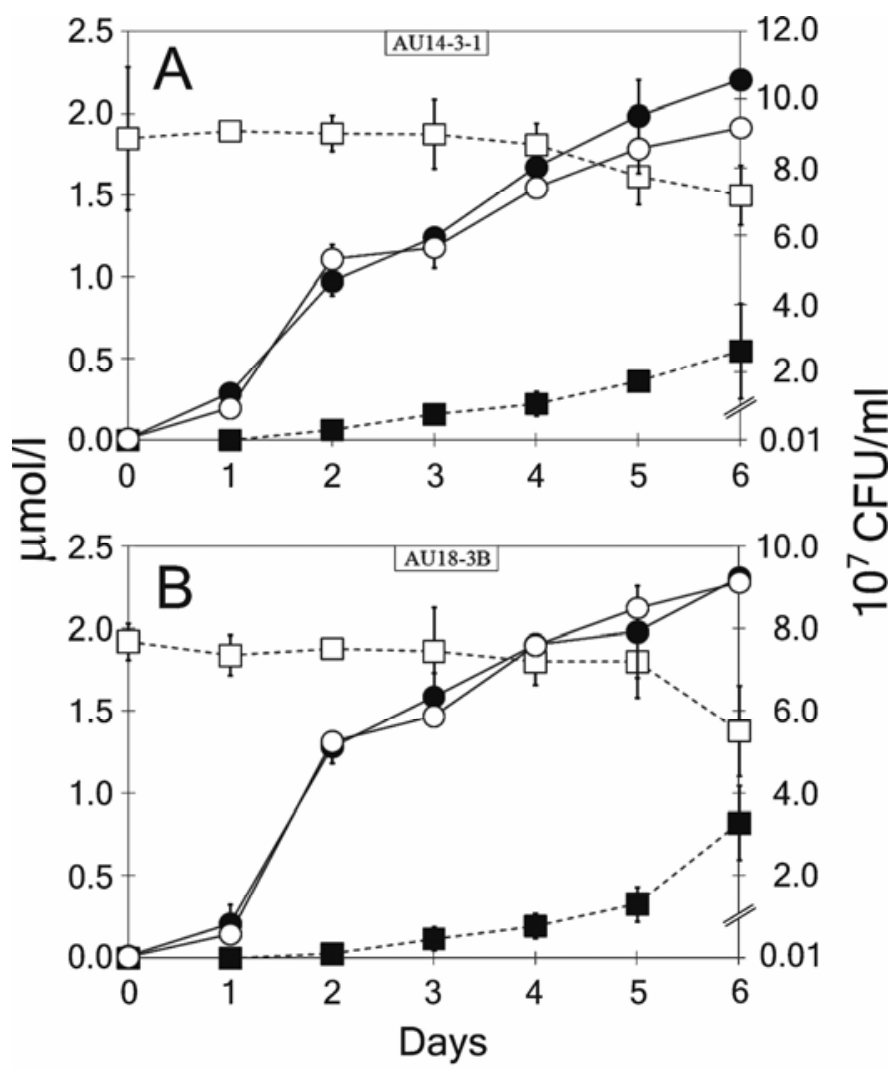

Fig. 6. Ochratoxin A (OTA) degradation and growth of Aureobasidium pullulans strains AU14-3-1 and AU18-3B during 6 days of incubation at $23^{\circ} \mathrm{C}$ in freshly prepared grape must. Dotted lines describe the levels of OTA ( $\square$ ) and ochratoxin $\alpha(\mathrm{OT} \alpha, \mathbf{\square})$ in the presence of A. pullulans strains A, AU14-31 and B, AU18-3B. Full lines describe the growth of the same A. pullulans strains same in the presence $(O)$ and in the absence $(-)$ of $2.0 \mu \mathrm{M}$ OTA. The initial OTA concentration $(2.0 \mu \mathrm{M})$ in the absence of the A. pullulans strains remained unchanged over the entire duration of the experiments. Values are the means \pm standard deviation ( $\mathrm{n}=9,3$ measurements in three experiments). the OTA concentrations were $1.50 \mu \mathrm{mol} / \mathrm{liter}$ for strain AU14-3-1 and $1.38 \mu \mathrm{mol} / \mathrm{liter}$ for strain AU18-3B, while the OT $\alpha$ concentrations were $0.55 \mu \mathrm{mol} / \mathrm{liter}$ for strain AU14-3-1 and 0.82 $\mu \mathrm{mol} / \mathrm{liter}$ for strain AU18-3B.

Biocontrol activity of $A$. pullulans strain LS30 in the vineyard. At harvest of growing season 2006, grape bunches treated with strain LS30 displayed much lower disease severity as compared to the untreated control (Fig. 7A). In particular, the biocontrol activity of strain LS30 caused a significant reduction $(81.9 \%)$ in the severity of Aspergillus rots. The disease incidence on grapes was reduced by $44.5 \%$ following applications with LS30, but this reduction was not statistically significant. The examination of wine grape samples revealed a prevalence of colonies belonging to Aspergillus species section Nigri, and the percentage of such colonies on samples of wine grape treated with strain LS30 was lower (45\% of reduction) than on samples from untreated control. During the growing season of 2007, no natural infections by Aspergillus spp. were recorded (data not shown).

Levels of OTA in wine grape samples from the vineyard. Analyses of OTA in wine grape samples from plots treated with LS30 showed that the yeast treatment significantly prevented contamination with OTA, when OTA levels were compared to those of the untreated control plots (Fig. 7B). No OTA was detected in LS30-treated wine grape samples. The levels of contamination in the untreated control samples ranged from 0.55 to $2.40 \mathrm{ng} / \mathrm{g}$ of berries. OT $\alpha$ was neither detected in LS30-treated nor in untreated wine grape samples.

\section{DISCUSSION}

The capability of different microorganisms to degrade mycotoxins is well documented. Species of Aspergillus, Penicillium, Botrytis, Alternaria, and Cladosporium, as well as protozoa and bacteria, are able to degrade OTA to the much less toxic compound OT $\alpha(1,6,29,60$, and Fig. 1). Likewise, patulin is converted to the less toxic compound ascladiol by the ascomycetous yeast Saccharomyces cerevisiae during alcoholic fermentation of cider, and in apple juice by the bacterium Gluconobacter oxydans, isolated from rotten apples $(48,54)$.

Microbial BCAs that have proven to be effective in controlling mycotoxigenic fungi have also proven to be able to degrade the respective mycotoxins in vitro. The biocontrol yeast Rhodotorula glutinis strain LS11 is effective in reducing infection by $P$. ex-
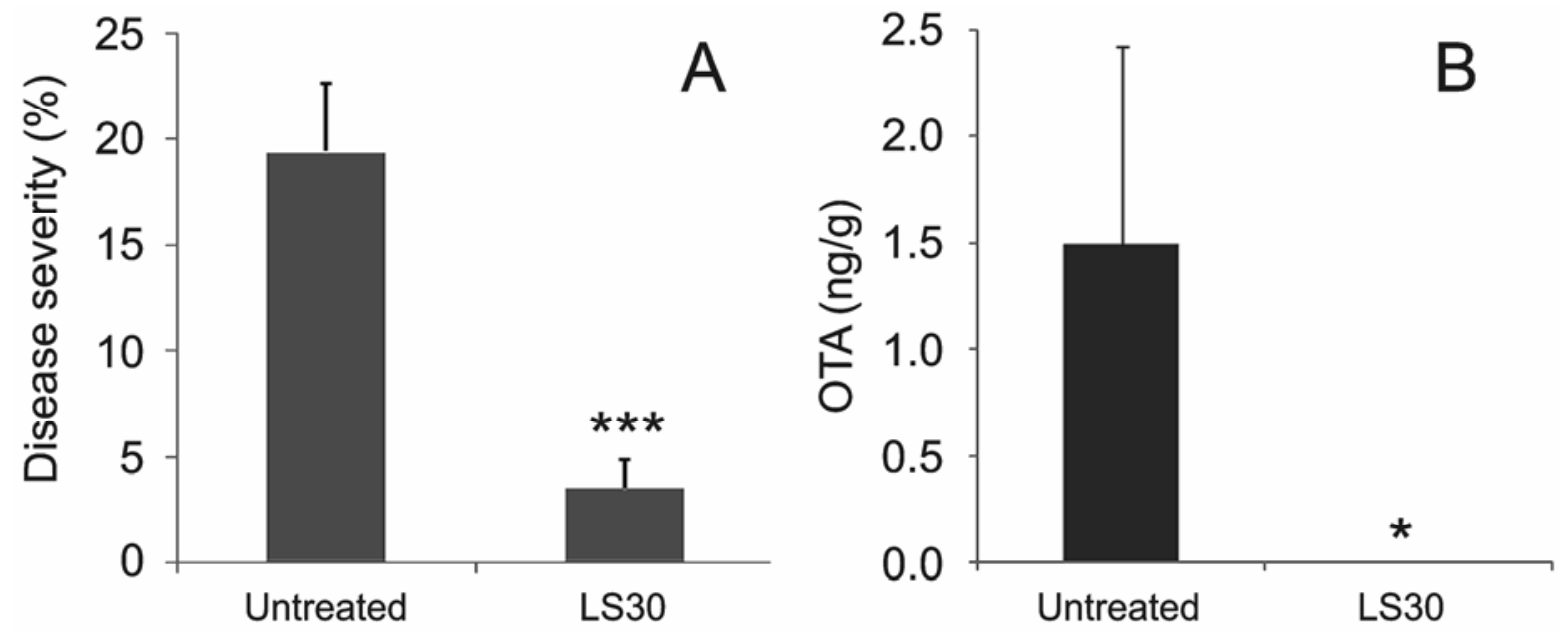

Fig. 7. A, Disease severity of Aspergillus rots and B, levels of ochratoxin A (OTA) on wine grape berries (cv. Montepulciano) pretreated with Aureobasidium pullulans strain LS30 in the vineyard, during the growing seasons of 2006. Disease severity was expressed as percentages \pm standard deviation of berries that showed symptoms of Aspergillus rots. Percentage values were converted into Bliss angular values prior to statistical analysis. OTA concentration was expressed as ng of toxin per gram of berries \pm standard deviation. The untreated control consisted of completely untreated plots. Values marked with $(*)$ and $(* * *)$ indicate significant differences at $P<0.05$ and 0.001 , respectively, according to the Student's $t$ test. 
pansum and patulin accumulation in infected apple fruit in storage (14). In addition, the same strain turns patulin to desoxypatulinic acid, a compound that is not considered to be toxic to microorganisms that are inhibited by patulin (16). Other biocontrol yeasts and yeastlike fungi such as Cryptococcus laurentii strain LS28 and Aureobasidium pullulans strain LS30 that control infection by $P$. expansum on stored apple fruits can degrade patulin in vitro, although at a lower rate than $R$. glutinis LS11 (14). Our preliminary experiments show that $A$. pullulans strains that control the ochratoxigenic pathogen A. carbonarius also degrade OTA in vitro (22). The above findings suggest that BCAs active against mycotoxigenic fungi have to cope with and resist mycotoxins, possibly through degradation of these compounds, and that mycotoxins could be involved in intermicrobial competition (17). There are no published reports on the degradation of OTA by microorganisms that are also active in biological control, nor are there reports on the influence of these microorganisms on OTA accumulation in wine grapes.

Strains of $A$. pullulans were reported to be effective BCAs, with antagonistic activity to different pathogens on several crops $(13,33,34,39,42)$. In LiBa medium, the growth of A. pullulans strains AU14-3-1, AU18-3B, AU34-2, and LS30 was not affected by the presence of OTA. OTA degradation by these strains was almost complete after 6 days. Biodegradation through the cleavage of the amide bond, linking OT $\alpha$ to L- $\beta$-phenylalanine in the molecule of OTA, appears to be the main means by which OTA decreases in the medium over time, since the micromolar concentrations of OT $\alpha$ on day 6 were about the same as those of OTA at the beginning of the experiments. The degradation of OTA by these strains of $A$. pullulans is similar to that achieved by other microorganisms that convert OTA to OT $\alpha$ and L- $\beta$-phenylalanine $(1,6,30,60$; Fig. 1), since these two products were also formed in our assays, as confirmed by LC-MS analyses. Furthermore, the adsorption of OTA to A. pullulans cells does not seem to contribute to the decrease of the mycotoxin in the growth medium, since only traces of OTA were detected by HPLC analyses of the cell extracts. Interestingly, the degradation kinetics of OTA in $\mathrm{LiBa}$ and grape must were similar. In both environments, the nutrient-poor $\mathrm{LiBa}$ and the nutrient-rich grape must, $A$. pullulans cells appear to start the degradation when they are in late logarithmic-early stationary phase of growth. It takes longer time for the cells to initiate degradation in grape must than in $\mathrm{LiBa}$, despite there being more CFU per milliliter in grape must at the onset of degradation. The starvation that the cells experience in $\mathrm{LiBa}$ could push them to degrade OTA earlier, in order to gain nutrients deriving from the degradation products. In addition, starvation itself could switch on a general stress response in the cells, which in turn could involve defense-related genes and processes, including the enzyme(s) responsible for OTA degradation.

The experimental conditions used to assess the biocontrol activity of the four strains of $A$. pullulans against $A$. carbonarius A1102 in laboratory-scale experiments are strongly favorable to the pathogen and to its production of OTA. In fact, OTA accumulation occurs mainly at ripening, when the fungus preferentially infects berries by entering skin wounds made by insects and/or injuries by meteorological phenomena (19). High levels of disease and of consequent contamination of wine grape with OTA take place when high humidity and temperature as well as damage to berries co-occur $(7,59)$. Furthermore, the levels of infection by $A$. carbonarius and the synthesis of OTA are the highest on wounded berries that are detached and that are subjected to the temperature and $\mathrm{RH}$ regimes that were used in our laboratoryscale experiments $(7,8,47)$. Although we had provided environmental conditions that were highly conducive to infection, three out of the four strains used provided an efficient protection of wine grape berries from $A$. carbonarius for up to 4 days after the beginning of these experiments. Although, by day 6 the level of protection decreased, berries treated with these same three strains still had significantly lower levels of infection (i.e., a lower count of infected wounds) than the untreated control berries. At this same time interval, the prevention of OTA accumulation in infected berries mirrored the biocontrol activity of the $A$. pullulans strains. The strains (AU14-3-1 and AU18-3B) that provided the best protection of berries from infection, also prevented mycotoxin accumulation in infected berries more efficiently than the other two strains (AU34-2 and LS30). On day 6, infected berries pretreated with AU14-3-1 and AU18-3B had the lowest OTA contamination even if their protective activity had diminished considerably compared with previous days. A. carbonarius and OTA can affect wine grape during the brief time interval (usually not exceeding 24 to $36 \mathrm{~h}$ ) that elapses from harvest to the beginning of the wine making process, when bunches detached from the living vine are more susceptible to fungal attack than when they are connected to the intact plant (7). AU14-3-1 and AU18-3B completely prevent infection of wounded berries up to day 2 . This suggests that these $A$. pullulans strains could prevent the attack by $A$. carbonarius on detached grape bunches also during the critical postharvest time interval immediately after harvest and before the wine-making process.

The A. pullulans strains used in this study survived on and colonized berries also in the cases when they were not able to protect them from infection by $A$. carbonarius. Similarly, strain LS11 of the biocontrol yeast $R$. glutinis was able to survive and proliferate in apples pretreated with this yeast and subsequently inoculated with $P$. expansum, even when it was not able to protect the fruits $(14,16)$.

The significant decrease of OTA contamination in infected berries pretreated with AU14-3-1 and AU18-3B could be due to an inhibition of growth of $A$. carbonarius. A similar situation was reported by Petersson et al. (49), who observed that Pichia anomala lowered the accumulation of OTA by inhibiting $P$. verrucosum, both in vitro and in artificially inoculated wheat. The decrease of OTA accumulation reflected an analogous decrease of growth of $P$. verrucosum, as measured by CFU counts (50). As it was also shown in the present study, A. carbonarius can produce both OTA and OT $\alpha$ in infected berries. This fungus produces OT $\alpha$ both as a biosynthetic precursor (26) and a degradation product of OTA $(1,6)$. Therefore, the low levels of OT $\alpha$ recorded in treated and untreated infected berries could be due to $A$. carbonarius. Although not significantly different from the infected untreated control berries, lower levels of OTA were also recorded in infected berries pretreated with strains AU34-2 and LS30, whereas the low levels of OT $\alpha$ recorded with all the four $A$. pullulans strains were not statistically different from each other and from the untreated control. In order to prove the inhibition of growth of $A$. carbonarius in infected berries pretreated with the $A$. pullulans strains (especially in the case of strains AU14-3-1 and AU18-3B), however, an unequivocal measurement of $A$. carbonarius biomass, possibly based on quantitative polymerase chain reaction (PCR), is needed. Nevertheless, the production of mycotoxins is not necessarily proportional to the biomass of the mycotoxigenic fungi, as was shown in studies on other mycotoxins (63). Although the overall amount of OTA accumulation is lower in A. carbonarius-infected berries pretreated with strains AU14-31 and AU18-3B of A. pullulans, an increase of OTA production per unit of pathogen biomass could take place as a consequence of competition among microorganisms for essential environmental factors. Intermicrobial competition is a stressful condition and is expected to have a dramatic effect on the secondary metabolism of spoilage fungi. Nutrient availability strongly affects mycotoxin production (44). In fact, when different $F u$ sarium species are co-inoculated on their host plant, the stress of the intermicrobial competition between them results in a higher mycotoxin production, as measured on a per unit of fungal biomass basis through real-time PCR, than when inoculated singly (63). 
Although the presence of OT $\alpha$ seems to be due to the activity of A. carbonarius, OTA degradation into OT $\alpha$ (or a specific inhibition of OTA biosynthesis) due to A. pullulans AU14-3-1 and AU18-3B cannot be ruled out. Physical contact between OTA and the cells of the BCAs or degrading enzyme(s) possibly secreted by these microorganisms is a prerequisite for toxin degradation. Since it is plausible that $A$. pullulans strains may grow only on the surface of infected berries, the physical contact could be favored by mycotoxin diffusion, which is greater in fruit with a lower content of structure-forming polysaccharides and looser texture (23), as in the case of grape berries infected by A. carbonarius. Results from wine-making experiments show that the majority of OTA is retained in pressed grape pomaces, i.e., in fractions which include skin residues along with the outer parts of berries (59). A. pullulans strains AU14-3-1 and AU18-3B were able to degrade exogenously-added OTA to OT $\alpha$ in grape must, which mimics the nutrient situation of grape berries. However, in infected berries pretreated with AU14-3-1 and AU18-3B, the source of OT $\alpha$ could not be ascertained because this metabolite is also present in A. carbonarius-infected control berries that are not pretreated with the A. pullulans strains, and the mycotoxigenic fungus itself could be a source of OT $\alpha(1,6,26)$. For the above reasons, the mechanism(s) underlying the decrease of OTA accumulation in infected berries pretreated with the strains AU14-3-1 and AU18-3B used in the laboratory-scale experiments of this study remains to be elucidated.

This study demonstrated that in laboratory-scale experiments strains of the ascomycetous yeastlike fungus A. pullulans degraded OTA to the less toxic compound OT $\alpha$ in synthetic medium and in grape must, can serve as effective biocontrol agents of $A$. carbonarius and cause a significant reduction of OTA contamination in berries infected by the fungal pathogen. Results from the field trial carried out during the growing season 2006 are encouraging. In this trial, the LS30 strain of A. pullulans appeared to be effective in the biocontrol of Aspergillus rots and in the prevention of OTA contamination in the vineyard. The LS30 strain had previously been shown to have biocontrol activity to different pathogens on a variety of crop species, including $A$. niger on stored table grapes $(13,43)$. Infection of grapes by Aspergillus was consistently less severe, following application of strain LS30, the population of potentially ochratoxigenic Aspergillus species section Nigri were also reduced in wine grape samples, and the accumulation of OTA was completely prevented. These results are in accordance with previous epidemiological investigations showing that Aspergillus section Nigri included the most common species occurring in Italian vineyards and that such species represent the main OTA-producing fungi $(3,4)$. OT $\alpha$ was not detected in samples from the vineyard probably because its concentration was below the limit of detection of the analytical method used in this study. Furthermore, OT $\alpha$ recorded in infected berries from laboratory-scale experiments represented only about 4 to $10 \%$ of OTA measured in the same samples.

The absence of natural Aspergillus infections on wine grape recorded in 2007 did not enable us to confirm the results obtained in 2006, and further investigations are needed. This is not surprising, since the occurrence of Aspergillus rot is closely related to specific meteorological conditions during the growing season. For detectable levels of disease and of consequent contamination of wine grape with OTA high humidity and temperature are needed $(7,59)$. As a consequence, it is difficult to obtain consistent field data from one year to another (40). However, our results encourage further research on microbial biocontrol agents as possible means for reducing mycotoxin contamination and as possible sources of detoxifying activity.

\section{ACKNOWLEDGMENTS}

This research was funded by the Italian Ministry of University and Scientific Research, Project PRIN no. 2005071422 "Biological and inte- grated control of Aspergillus carbonarius: effectiveness on ochratoxin A content and grape-wine chain". We thank V. M. T. Lattanzio for LC-MS analyses for confirmation of ochratoxin $\alpha$ and L- $\beta$-phenylalanine, S. A. Ingela Wright for critical reading and language editing of the manuscript, and L. Filippini from ISAGRO for his invaluable support.

\section{LITERATURE CITED}

1. Abrunhosa, L., Serra, R., and Venâncio, A. 2002. Biodegradation of Ochratoxin A by fungi isolated from grapes. J. Agric. Food Chem. 50:7493-7496

2. Avantaggiato, G., Solfrizzo, M., and Visconti, A. 2005. Recent advances on the use of adsorbent materials for detoxification of Fusarium mycotoxins. Food Addit. Contam. 22:379-388.

3. Battilani, P., Giorni, P., and Pietri, A. 2003. Epidemiology of toxinproducing fungi and ochratoxin A occurrence in grape. Eur. J. Plant Pathol. 109:715-722.

4. Battilani, P., and Pietri, A. 2002. Ochratoxin A in grapes and wine. Eur. J. Plant Pathol. 108:639-643.

5. Bejaoui, H., Mathieu, F., Taillandier, P., and Lebrihi, A. 2004. Ochratoxin A removal in synthetic and natural grape juices by selected oenological Saccharomyces strains. J. Appl. Microbiol. 97:1038-1044.

6. Bejaoui, H., Mathieu, F., Taillandier, P., and Lebrihi, A. 2006. Biodegradation of ochratoxin A by Aspergillus section Nigri species isolated from French grapes: A potential means of ochratoxin A decontamination in grape juices and musts. FEMS Microbiol. Lett. 255:203-208.

7. Bellí, N., Marìn, S., Coronas, I., Sanchis, V., and Ramos, A. J. 2007. Skin damage, high temperature and relative humidity as detrimental factors for Aspergillus carbonarius infection and ochratoxin A production in grapes. Food Control 18:1343-1349.

8. Bellí, N., Ramos, A. J., Sanchis, V., and Marìn, S. 2004. Incubation time and water activity effects on ochratoxin A production by Aspergillus section Nigri strains isolated from grapes. Lett. Appl. Microbiol. 38:7277.

9. Bennett, J. W., and Klich, M. 2003. Mycotoxins. Clin. Microbiol. Rev. $16: 497-516$

10. Breitholtz-Emanuelsson, A., Fuchs, R., Hult, K., and Appelgren, L. E. 1992. Syntheses of ${ }^{14} \mathrm{C}$-ochratoxin A and ${ }^{14} \mathrm{C}$-ochratoxin B and a comparative study of their distribution in rats using whole body autoradiography. Pharmacol. Toxicol. 70:255-261.

11. Breitholtz-Emanuelsson, A., Minervini, F., Hult, K., and Visconti, A. 1994. Ochratoxin A in human serum samples collected in southern Italy from healthy individuals and individuals suffering from different kidney disorders. Nat. Toxins 6:366-370.

12. Castellari, M., Versari, A., Fabiani, A., Parpinello, G. P., and Galassi, S. 2001. Removal of ochratoxin A in red wines by means of adsorption treatments with commercial fining agents. J. Agric. Food Chem. 49:39173921.

13. Castoria, R., De Curtis, F., Lima, G., Caputo, L., Pacifico, S., and De Cicco, V. 2001. Aureobasidium pullulans (LS30) an antagonist of postharvest pathogens of fruits: study on its modes of action. Postharvest Biol. Technol. 22:7-17.

14. Castoria, R., Morena, V., Caputo, L., Panfili, G., De Curtis, F., and De Cicco, V. 2005. Effect of the biocontrol yeast Rhodotorula glutinis strain LS11 on patulin accumulation in stored apples. Phytopathology 95:12711278.

15. Castoria, R., and Logrieco, A. 2007. Mycotoxins in fruits and fruit-derived products-An overview. In: Microbial Biotechnology in Horticulture, Vol. 2. R. C. Ray and O. P. Ward, eds. Science Publishers, Inc., Enfield, NH.

16. Castoria, R., Mannina, L., Maiuro, L., Sobolev, A., Ritieni, A., Ferracane, R., and Spina, A. 2007. Nuovi dati sul decremento di patulina causato da un lievito di biocontrollo in mele infettate da Penicillium expansum. Rapporti ISTISAN (Istituto Superiore di Sanità, Rome, Italy) 07-37:147151.

17. Castoria, R., Wright, S. A. I., and Droby, S. 2008. Biological Control of Mycotoxigenic Fungi in Fruits. Pages 311-333 in: Mycotoxins in Fruits and Vegetables. R. Barkai-Golan and N. Paster, eds. Elsevier, San Diego, CA.

18. Commission Regulation (EC) No $123 / 2005$ of 26 January 2005. Official Journal of the European Union. L25/3-25/5.

19. Cozzi, G., Pascale, M., Perrone, G., Visconti, A., and Logrieco, A. 2006. Effect of Lobesia botrana damages on black aspergilli rot and ochratoxin A content in grapes. Int. J. Food. Microbiol. 111(suppl.):S88-92.

20. Creppy, E. E., Stormer, F. C., Röschenthler, R., and Dirheimer, G. 1983. Effects of two metabolites of ochratoxin A (4R)-4-hydroxyochratoxin A and ochratoxin $\alpha$ on immune response in mice. Infect. Immun. 39:10151018.

21. De Curtis, F., Caputo, L., Castoria, R., Lima, G., Stea, G., and De Cicco, V. 2004. Use of fluorescent amplified fragment length polymorphism 
(fAFLP) to identify specific molecular markers for the biocontrol agent Aureobasidium pullulans strain LS30. Postharvest Biol. Technol. 34:179186.

22. de Felice, D. V., Solfrizzo, M., de Curtis, F., Visconti, A., De Cicco, V., and Castoria. R. 2006. Aureobasidium pullulans strains degrade Ochratoxin A in vitro and decrease infections by Aspergillus carbonarius on wine grape. Int. Organ. Biol. Integrated Control Noxious Animals Plants/West Paleartic Reg. Sect. Bull. Vol. 30(6):38.

23. Drusch, S., and Ragab, W. 2003. Mycotoxins in fruits, fruit juices, and dried fruits. J. Food Prot. 66:1514-1527.

24. Föllmann, W., Hillebrand, I. E., Creppy, E. E., and Bolt, H. M. 1995. Sister chromatid exchange frequency in cultured isolated porcine urinary bladder epithelial cells (PUBEC) treated with ochratoxin A and alpha. Arch. Toxicol. 69:280-286.

25. Galtier, P., and Alvinerie, M. 1976. In vitro transformation of ochratoxin A by animal microbial floras. Ann. Rech. Vet. 7:91-98.

26. Harris, J. P., and Mantle, P. G. 2001. Biosynthesis of ochratoxins by Aspergillus ochraceus. Phytochemistry 58:709-716.

27. Harwig, J., Kuiper-Goodman, T., and Scott, P. M. 1983. Microbial food toxicants: Ochratoxins. Pages 193-238 in: Handbook of Foodborne Diseases of Biological Origin. M. Rechcigl, ed. CRC Press, Boca Raton, FL.

28. Hassen, W., Abid, S., Achour, A., Creppy, E., and Hassen, B. 2004. Ochratoxin $A$ and $\beta_{2}$-microglobulinuria in healthy individuals and in chronic interstitial nephropathy patients in the centre of Tunisia: A hot spot of ochratoxin A exposure. Toxicology 199:185-193.

29. Hult, K., Plestina, R., and Habazin Novak, V. 1982. Ochratoxin A in human blood and Balkan endemic nephropathy. Arch. Toxicol. 51:313321.

30. Hult, K., Teiling, A., and Gatenbeck, S. 1976. Degradation of ochratoxin A by a ruminant. Appl. Environ. Microbiol. 32:443-444.

31. Hwang, C. A., and Draughon, F. A. 1994. Degradation of ochratoxin A by Acinetobacter calcoaceticus. J. Food Prot. 57:410-414.

32. International Agency For Research On Cancer. 1993. Some Naturally Occurring Substances: Food Items and Constituents, Heterocyclic Aromatic Amines and Mycotoxins. Pages 489-520 in: IARC Monographs on the Evaluation of the Carcinogenic Risk of Chemicals to Man, Vol. 56. IARC, Lyon, France.

33. Ippolito, A., Schena, L., Pentimone, I., and Nigro, F. 2005. Control of postharvest rots of sweet cherries by pre- and postharvest applications of Aureobasidium pullulans in combination with calcium chloride or sodium bicarbonate. Postharvest Biol. Technol. 36:245-252.

34. Janisiewicz, W. J., Tworkoski, T. J., and Sharer, C. 2000. Characterizing the mechanism of biological control of postharvest diseases on fruits with a simple method to study competition for nutrients. Phytopathology 90:1196-1200.

35. Jonsyn, F. E., Maxwell, S. M., and Hendrickse, R. G. 1995. Ochratoxin A and aflatoxins in breast milk samples from Sierra Leone. Mycopathologia 131:121-126.

36. Karlovsky, P. 1999. Biological detoxification of fungal toxins and its use in plant breeding, feed and food production. Nat. Toxins 7:1-23.

37. King, A. D., Jr., Hocking, A. D., and Pitt, J. I. 1979. Dichloran-rose Bengal medium for enumeration and isolation of molds from foods. Appl. Environ. Microbiol. 37:959-964.

38. Krogh, P., Axelsen, N. H., Elling, F., Gyrd-Hansen, N., Hald, B., Hyldgaard-Jensen, J., Larsen, A. E., Madsen, A., Mortensen, H. P., Moller, T., Petersen, O. K., Ravnskov, U., Rostgaard, M., Aalund, O. 1974. Experimental porcine nephropathy. Changes of renal function and structure induced by ochratoxin A- contaminated feed. Acta Pathol. Microbiol. Immunol. A 246:1-21.

39. Leibinger, W., Breuker, B., Hahn, M., and Mendgen, K. 1997. Control of postharvest pathogens and colonization of the apple surface by antagonistic microorganisms in the field. Phytopathology 87:1103-1110.

40. Leong, S. L., Hocking, A. D., Varelis, P., Giannikopoulos, G., and Scott, E. S. 2006. Fate of Ochratoxin A during Vinification of Semillon and Shiraz Grapes. J. Agric. Food Chem. 54:6460-6464.

41. Li, S., Marquardt, R. R., Frohlich, A. A., Vitti, T. G., and Crow, G. 1997. Pharmacokinetics of ochratoxin A and its metabolites in rats. Toxicol. Appl. Pharm. 145:82-90.

42. Lilly, V. G., and Barnett, H. L. 1951. Physiology of the Fungi. McGrawHill, NY.
43. Lima, G., Ippolito, A., Nigro, F., and Salerno, M. 1997. Effectiveness of Aureobasidium pullulans and Candida oleophila against postharvest strawberry rots. Postharvest Biol. Technol. 10:169-178.

44. Luchese, R. H., and Harrigan, W. F. 1993. Biosynthesis of aflatoxin - the role of nutrition factors. J. Appl. Bacteriol. 74:5-14.

45. McGrath, M. J., and Andrews, J. H. 2006. Temporal Changes in Microscale Colonization of the Phylloplane by Aureobasidium pullulans. Appl. Environ. Microbiol. 72:6234-6241.

46. Micco, C., Ambruzzi, M. A., Miraglia, M., Brera, C., Onori, R., and Benelli, L. 1991. Contamination of human milk with ochratoxin A. IARC Sci. Publ. 115:105-108.

47. Mitchell, D., Parra, R., Aldred, D., and Magan, N. 2004. Water and temperature relations of growth and ochratoxin A production by Aspergillus carbonarius strains from grapes in Europe and Israel. J. Appl. Microbiol. 97:439-445.

48. Moss, M. O., and Long, M. T. 2002. Fate of patulin in the presence of yeast Saccharomices cerevisiae. Food Addit. Contam. 19:387-399.

49. Pettersson, H., Kiessling, K. H., and Ciszuk, P. 1982. Degradation of ochratoxin A in rumen. Pages 313-316 in: Proceedings of the V International IUPAC Symposium Mycotoxins Phycotoxins. Austrian Chemical Society, Austria, Vienna.

50. Petersson, S., Wittrup Hansen, M., Axberg, K., Hult, K., and Schnurer, J. 1998. Ochratoxin A accumulation in cultures of Penicillium verrucosum with the antagonistic yeast Pichia anomala and Saccharomyces cerevisiae. Mycol. Res. 102:1003-1008.

51. Pfohl-Leszkowicz, A., Petkova-Bocharova, T., Chernozemsky, I. N., and Castegnaro, M. 2002. Balkan endemic nephropathy and associated urinary tract tumours: A review on aetiological causes and the potential role of mycotoxins. Food Addit. Contam. 19:282-302.

52. Prakitchaiwattana, C. J., Fleet, G. H., and Heard, G. M. 2004. Application and evaluation of denaturing gradient gel electrophoresis to analyse the yeast ecology of wine grapes. FEMS Yeast Res. 4:865-877.

53. Raper, K. B., and Fennell, D. I. 1965. The Genus Aspergillus. R. E. Krieger Publishing Company, Malabar, FL.

54. Ricelli, A., Baruzzi, F., Solfrizzo, M., Morea, M., and Fanizzi, F. P. 2007. Biotransformation of patulin by Gluconobacter oxydans. Appl. Environ. Microbiol. 73:785-792.

55. Ryu, D., and Bullerman, L. B. 1999. Effect of cycling temperatures on the production of deoxynivalenol and zearalenone by Fusarium graminearum. J. Food Prot. 62:1451-1455.

56. Scott, P. M. 2005. Biomarker of human exposure to ochratoxin A. Food Addit. Contam. 1:99-107.

57. Takesako, K., Ikai, K., Haruna, F., Endo, M., Shimanaka, K., Sono, E., Nakamura, T., Kato, I., and Yamaguchi, H. 1991. Aureobasidins, new antifungal antibiotics. Taxonomy, fermentation, isolation, and properties. J. Antibiot. 44:919-924.

58. Varga, J., Rigó, K., and Téren, J. 2000. Degradation of ochratoxin A by Aspergillus species. Int. J. Food Microbiol. 59:1-7.

59. Visconti, A., Perrone, G., Cozzi, G., and Solfrizzo, M. 2008. Managing ochratoxin A risk in the grape-wine food chain. Food Addit. Contam. 25:193-202.

60. Wegst, W., and Lingens, F. 1983. Bacterial degradation of ochratoxin A. FEMS Microbiol. Lett. 17:341-344.

61. West, T. P., and Strohfus, B. 2001. Polysaccharide production by a reduced pigmentation mutant of Aureobasidium pullulans NYS-1. Lett. Appl. Microbiol. 33:169-172.

62. Xiao, H., Madhyastha, S., Marquardt, R. R., Li, S., Vodela, J. K. Frohlich, A. A., and Kemppainen, B. W. 1996. Toxicity of ochratoxin A, its opened lactone form and several of its analogs: Structure-activity relationships. Toxicol. Appl. Pharm. 137:182-192.

63. Xu, X. M., Monger, W., Ritieni, A., and Nicholson, P. 2007. Effect of temperature and duration of wetness during initial infection periods on disease development, fungal biomass and mycotoxin concentrations on wheat inoculated with single, or combinations of, Fusarium species. Plant Pathol. 56:943-956.

64. Yamazaki, M., Suzuki, S., Sakakibara, Y., and Miyaki, K. 1971. The toxicity of 5-chloro- 8-hydroxy- 3,4-dihydro- 3-methyl -isocoumarin-7carboxylic acid, a hydrolyzate of ochratoxin A. Jpn. J. Med. Sci. Biol. 24:245-250.

65. Zimmerli, B., and Dick, R. 1996. Ochratoxin A in table wine and grapejuice: Occurrence and risk assessment. Food Addit. Contam. 13:655-668. 